Annals of Pure and Applied Mathematics

Vol.19, No.2, 2019, 127-139

ISSN: 2279-087X (P), 2279-0888(online)

Published on 5 April 2019

Annals of

www.researchmathsci.org

DOI: http://dx.doi.org/10.22457/apam.581v19n2a1

Pure and Applied

Mathematics

\title{
On Normal Sub-Intuitionistic Fuzzy Multigroups
}

\author{
I.M.Adamu ${ }^{1}$, Y.Tella ${ }^{2}$ and A.J.Alkali ${ }^{3}$
}

${ }^{1}$ Department of Mathematics, Federal University Dutse

P.M.B. 7156 Dutse, Jigawa State, Nigeria

Email: idreesmuhammadadam@gmail.com

${ }^{2}$ Department of Mathematical Sciences, Kaduna State University-Kaduna

P.M.B. 2339 Kaduna State, Nigeria

Email: yot2002ng@yahoo.com

${ }^{3}$ Department of Mathematics, Ahmadu Bello University Zaria

P.M.B. 1094 Samaru-Zaria, Kaduna State, Nigeria

Email: alkali4real2012@gmail.com

Received 16 February 2019; accepted 1 April 2019

\begin{abstract}
This paper developed the concept of normal sub-intuitionistic fuzzy Multigroups. It then established that intersection, union, of any two normal subintuitionistic fuzzy multigroups is also normal. The inverse of any normal subintuitionistic fuzzy multigroup is normal and for any normal sub-intuitionistic fuzzy multigroup, the root (support) set of normal sub-intuitionistic fuzzy multigroup is a normal sub-intuitionistic fuzzy group. It also showed that under the isomorphism function between any two groups, the image of a normal sub-intuitionistic fuzzy multigroup under the isomorphism is a normal sub-intuitionistic fuzzy multigroup and the inverse image of a normal sub-intuitionistic fuzzy multigroup under the isomorphism is a normal subintuitionistic fuzzy multigroup.
\end{abstract}

Keywords: Multiset, fuzzy multiset, intuitionistic fuzzy multiset, multi groups, fuzzy multigroups, intuitionistic fuzzy multi group, isomorphism function, normal subintuitionistic fuzzy multigroup

\section{AMS Mathematics Subject Classification (2010): 08A72}

\section{Introduction}

Classical set theory introduced by George Cantor has proved itself to be one of the most powerful tools of modern Mathematics. In this set theory, a set is a well-defined collection of distinct objects. If repeated occurrences of any object are allowed in a set, then the mathematical structure is called as multiset. Thus, a multiset differs from a set in the sense that each element has a multiplicity. An account of the development of multiset theory can be seen in $[1,2,3,4]$. Most of the real life situations are complex and for modeling them we need a simplification of the complex system. The simplification must be in such a way that the information lost should be minimum. One way to do this is to allow some degree of uncertainty into it. To handle situations like this, many tools were suggested. They include fuzzy sets, rough sets, soft sets etc. Considering the uncertainty 


\section{I.M.Adamu, Y.Tella and A.J.Alkali}

factor, Zadeh [5] introduced Fuzzy sets in 1965, in which a membership function assigns to each element of the universe of discourse, a number from the unit interval $[0,1]$ to indicate the degree of belongingness to the set under consideration. Fuzzy sets were introduced with a view to reconcile mathematical modeling and human knowledge in the engineering sciences. Since then, a considerable body of literature has blossomed around the concept of fuzzy sets in an incredibly wide range of areas, from mathematics and logics to traditional and advanced engineering methodologies. In 1983, Atanassov [6, 7] introduced the concept of Intuitionistic Fuzzy sets. The same time a theory called 'Intuitionistic Fuzzy set theory' was independently introduced by Takeuti and Titani [8] as a theory developed in (a kind of) Intuitionistic logic. An Intuitionistic Fuzzy set is characterized by two functions expressing the degree of membership and the degree of nonmembership of elements of the universe to the Intuitionistic Fuzzy set. Among the various notions of higher-order Fuzzy sets, Intuitionistic Fuzzy sets proposed by Atanassov provide a flexible framework to explain uncertainty and vagueness. It is wellknown that in the beginning of the last century L.Brouwer introduced the concept of Intuitionism. The name Intuitionistic Fuzzy set is due to George Gargove, with themotivation that their fuzzification denies the law of excluded middle-one of the main ideas of Intuitionism. As a generalization of multiset, Yager [9] introduced fuzzy multisets and suggested possible applications to relational databases. An element of a Fuzzy Multiset can occur more than once with possibly the same or different membership values. The concept of Intuitionistic Fuzzy Multiset is introduced in [10] which have applications in medical diagnosis and robotics. In mathematics, abstract algebra is the study of algebraic structures and more specifically the term algebraic structure generally refers to a set (called carrier set or underlying set) with one or more finitely operations defined on it. Examples of algebraic structures include groups, rings, fields, and lattices. The algebraic structures of Fuzzy multisets are introduced in [11]. Adamu et al. [12] developed the concepts of normal and soft normal groups under multisets and soft multisets context. They defined the concepts of normal submultigroups and soft normal Multigroups and proved some of their related algebraic structures. In this paper we are extending these algebraic structures on intuitionistic fuzzy multisets and intuitionistic fuzzy multigroups by introducing a new concept named normal sub intuitionistic fuzzy multigroups.

\section{Preliminaries}

Definition 2.1. [13] Let $X$ be a set. A multiset (mset) $M$ drawn from $X$ is represented by a function count $M$ or $C_{M}$ defined as $C_{M}: X \rightarrow\{0,1,2,3, \ldots\}$. For each $x \in X, C_{M}(x)$ is the characteristic value of $x$ in $M$. Here $C_{M}(x)$ denotes the number of occurrences of $x$ in $M$.

Definition 2.2. [14] Let $X$ be a group. A multi set $G$ over $X$ is a multi-group over $X$ if the count of $G$ satisfies the following two conditions.

i. $\quad C_{G}(x y) \geq C_{G}(x) \wedge C_{G}(y) \forall x, y \in X$

ii. $C_{G}\left(x^{-1}\right) \geq C_{G}(x) \forall x \in X$.

Definition 2.3. [15] If $X$ is a collection of objects, then a fuzzy set $A$ in $X$ is a set of ordered pairs: $A=\left\{\left(x, \mu_{A}(x)\right): x \in X, \mu_{A}: X \rightarrow[0,1]\right\}$ where $\mu_{A}$ is called the membership function of $A$, and is defined from $X$ into $[0,1]$. 


\section{On Normal Sub-Intuitionistic Fuzzy Multigroups}

Definition 2.4. [16] Let $G$ be a group and $\mu \in F P(G)$ (fuzzy power set of $G$ ), then $\mu$ is called fuzzy subgroup of $G$ if

$$
\begin{array}{ll}
\text { i. } & \mu(x y) \geq \mu(x) \wedge \mu(y) \forall x, y \in X \text { and } \\
\text { ii. } & \mu\left(x^{-1}\right) \geq \mu(x) \forall x \in X .
\end{array}
$$

Definition 2.5. [10] Let $X$ be a nonempty set. An Intuitionistic Fuzzy Multiset $A$ denoted by IFMS drawn from $X$ is characterized by two functions : 'count membership' of $A\left(C M_{A}\right)$ and 'count non membership' of $A\left(C N_{A}\right)$ given respectively by $C M_{A}: X \rightarrow Q$ and $C N_{A}: X \rightarrow Q$ where $Q$ is the set of all crisp multisets drawn from the unit interval $[0,1]$ such that for each $x \in X$, the membership sequence is defined as a decreasingly ordered sequence of elements in $A\left(C M_{A}\right)$ which is denoted by $\left(\mu_{A}^{1}(x), \mu_{A}^{2}(x), \ldots, \mu_{A}^{p}(x)\right)$ where $\left(\mu_{A}^{1}(x) \geq \mu_{A}^{2}(x) \geq, \ldots, \mu_{A}^{p}(x)\right)$ and the corresponding non membership sequence will be denoted by

$\left(v_{A}^{1}(x), v_{A}^{2}(x), \ldots, v_{A}^{p}(x)\right)$ such that $0<\mu_{A}^{i}(x)+v_{A}^{i}(x)<1$ for every $x \in X$ and $i=1,2, \ldots, p$.

An IFMS $A$ is denoted by

$$
A=\left\{<x:\left(\mu_{A}^{1}(x), \mu_{A}^{2}(x), \ldots, \mu_{A}^{p}(x)\right),\left(v_{A}^{1}(x), v_{A}^{2}(x), \ldots, v_{A}^{p}(x)\right)>: x \in X\right\}
$$

Definition 2.6. [10] Length of an element $x$ in an IFMS $A$ is defined as the Cardinality of $C M_{A}(x)$ or $C N_{A}(x)$ for which $0<\mu_{A}^{i}(x)+v_{A}^{i}(x)<1$ and it is denoted by $L(x: A)$. That is

$$
\begin{gathered}
L(x: A)=\left|C_{A}(x)\right|=\left|C_{A}(x)\right| \\
L(x: A)=|C M A(x)|=|C N A(x)|
\end{gathered}
$$

Definition 2.7. [10] If $A$ and $B$ are IFMSs drawn from $X$ then

$$
L(x: A, B)=\operatorname{Max}\{L(x: A), L(x: B)\} \text {. }
$$

Alternatively we use $L(x)$ for $L(x: A, B)$.

Definition 2.8. [11] For any two IFMSs $A$ and $B$ drawn from a set $X$, the following operations and relations will hold. Let

$$
\begin{aligned}
A & =\left\{<x:\left(\mu_{A}^{1}(x), \mu_{A}^{2}(x), \ldots, \mu_{A}^{p}(x)\right),\left(v_{A}^{1}(x), v_{A}^{2}(x), \ldots, v_{A}^{p}(x)\right)>: x \in X\right\} \\
\text { And } & \\
B & =\left\{<x:\left(\mu_{A}^{1}(x), \mu_{A}^{2}(x), \ldots, \mu_{A}^{p}(x)\right),\left(v_{A}^{1}(x), v_{A}^{2}(x), \ldots, v_{A}^{p}(x)\right)>: x \in X\right\}
\end{aligned}
$$

Then

i. Inclusion

$A \subset B \Leftrightarrow \mu_{A}^{j}(x) \leq \mu_{A}^{j}(x)$ and $v_{A}^{j}(x)+v_{A}^{j}(x) ; j=1,2, \ldots, L(x), x \in X$

$A=B \Leftrightarrow A \subset B$ and $B \subset A$

ii. Complement

$\neg A=\left\{<x:\left(v_{A}^{1}(x), v_{A}^{2}(x), \ldots, v_{A}^{p}(x)\right),\left(\mu_{A}^{1}(x), \mu_{A}^{2}(x), \ldots, \mu_{A}^{p}(x)\right)>: x \in X\right\}$

iii. Union $(A \cup B)$

In $A \cup B$ the membership and non-membership values are obtained as follows.

$$
\mu_{A \cup B}^{j}(x)=\mu_{A}^{j}(x) \vee \mu_{B}^{j}(x)
$$


I.M.Adamu, Y.Tella and A.J.Alkali

$$
v_{A \cup B}^{j}(x)=v_{A}^{j}(x) \wedge v_{A}^{j}(x), j=1,2, \ldots, L(x), x \in X .
$$

iv. Intersection $(A \cap B)$

In $A \cap B$ the membership and non-membership values are obtained as follows.

$$
\begin{array}{r}
\mu_{A \cap B}^{j}(x)=\mu_{A}^{j}(x) \wedge \mu_{B}^{j}(x) \\
v_{A \cap B}^{j}(x)=v_{A}^{j}(x) \vee v_{A}^{j}(x), j=1,2, \ldots, L(x), x \in X .
\end{array}
$$

Definition 2.9. [11] If $X$ and $Y$ are two nonempty sets and $f: X \rightarrow Y$ be a mapping. Then

i. The image of the FMS $A \in F M(X)$ under the mapping $f$ is denoted by $f(A)$, or

$$
C M_{f[A]}(y)=\left\{\begin{array}{cc}
\mathrm{V}_{f(x)=y} C M_{A}(x) & \text { if } f^{-1}(y) \neq \phi \\
0 & \text { otherwise }
\end{array}\right.
$$

ii. The inverse image of the FMS $B \in F M(Y)$ under the mapping $f$ is denoted by $f^{-1}(B)$ or $f^{-1}[B]$, where $C M_{f^{-1}[B]}(x)=C M_{B} f[x]$.

Definition 2.10. [11] Let $X$ be a group. A fuzzy multiset $G$ over $X$ is a fuzzy multi group (FMG) over $X$ if the count (count membership) of $G$ satisfies the following two conditions.

i. $\quad C M_{G}(x y) \geq C M_{G}(x) \wedge C M_{G}(y) \forall x, y \in X$.

ii. $\quad C M_{G}\left(x^{-1}\right)=C M_{G}(x) \forall x \in X$

Definition 2.11. [12] A submgroup $H$ of a mgroup $M \in M G(X)$ is said to be a normal submgroup iff for any $h \in H^{*}$,

$$
C_{H}\left(x^{-1} h x\right) \geq C_{H}(h), \forall x \in M^{*} .
$$

Theorem 2.12. [12] Let $M_{1}$ and $M_{2}$ be submgroups of a mgroup $M \in M G(X)$ such that $M_{1}, M_{2} \in \Delta \wp(M)$, then

i. $\quad M_{1} \cap M_{2} \in \Delta \wp(M)$

ii. $\quad M_{1} \cup M_{2} \in \Delta \wp(M)$.

Theorem 2.13 [12] If $H \in \Delta \wp(M)$ then $H^{*}$ is a normal subgroup of $M^{*}$

Theorem 2.14. [12] Let $X$ be a group and $M \in M G(X)$. If $M \in \Delta \wp(M)$, then $M^{-1} \in \Delta \wp(M)$.

Theorem 2.15. [12] Let $X, Y$ be two groups and $f: X \rightarrow Y$ be an isomorphism of groups. Suppose $M_{1} \in M G(X), M_{2} \in M G(Y)$ and $f\left(M_{1}\right) \subseteq M_{2}$

i. $\quad$ if $H \in \Delta \wp\left(M_{1}\right)$, then $f(H) \in \Delta \wp\left(M_{2}\right)$;

ii. if $H \in \Delta \wp\left(M_{2}\right)$ then $f^{-1}(H) \in \Delta \wp\left(M_{1}\right)$.

Definition 2.16. [17] Let $X$ be a group. An intuitionistic fuzzy multiset $G$ over $X$ is an intuitionistic fuzzy multi group (IFMG) over $X$ if the counts (count membership and nonmembership) of $G$ satisfies the following two conditions.

i. $\quad C M_{G}(x y) \geq C M_{G}(x) \wedge C M_{G}(y) \forall x, y \in X$. 
On Normal Sub-Intuitionistic Fuzzy Multigroups

ii. $\quad C M_{G}\left(x^{-1}\right) \geq C M_{G}(x) \forall x \in X$.

iii. $\quad C N_{G}(x y) \leq C M_{G}(x) \wedge C M_{G}(y) \forall x, y \in X$.

iv. $\quad C N_{G}\left(x^{-1}\right) \leq C M_{G}(x) \forall x \in X$.

Definition 2.17. [17] Let $G \in F M S(X)$. Then define

$G^{*}=\left\{x \in X: C M_{G}(x)=C M_{G}(e)\right.$ and $\left.C N_{G}(x)=C N_{G}(e)\right\}$.

Proposition 2.18. [17] Let $G \in F M G(X)$. Then $G^{*}$ is a subgroup of $X$.

Definition 2.19. [17] Let $G \in F M S(X)$. Let $j \in \mathbb{N}$. Then define

$$
G^{j}=\left\{x \in X: \mu_{G}^{j}(x) \geq 0, \mu_{G}^{j+1}(x)=0 \text { and } v_{G}^{j}(x)=0\right\} \text {. }
$$

Theorem 2.20. [17] Let $G \in F M G(X)$. Then $G^{j}$ is a subgroup of $X$ iff

$$
\mu_{G}^{j+1}\left(x y^{-1}\right)=0 \text { and } v_{G}^{j+1}\left(x y^{-1}\right)=0 \forall x, y \in G^{j}
$$

\section{Normal sub-intuitionistic fuzzy multigroups}

Throughout this section, let $X$ be a group with binary operation and the identity element is $e$. Also we assume that the intuitionistic fuzzy multisets and intuitionistic fuzzy Multigroups are taken from $\operatorname{IFMS}(X)$ and $\operatorname{IFMG}(X)$ respectively.

Definition 3.1. A sub-intuitionistic fuzzy multigroup $H$ of an intuitionistic fuzzy multigroup $G \in \operatorname{IFMG}(X)$ is said to be a normal sub-intuitionistic fuzzy Multigroups iff for any $h \in H^{*}$,

i. $\quad C M_{H}\left(x^{-1} h x\right) \geq C M_{H}(h), \forall x \in G^{*}$.

ii. $\quad C N_{H}\left(x^{-1} h x\right) \leq C N_{H}(h) \forall x \in G^{*}$.

We denote the set of all normal sub-intuitionistic fuzzy Multigroups of an intuitionistic fuzzymgroup $G \in \operatorname{IFM} G(X)$ by $\triangle \wp S I F M G(G)$ and the normality of $H$ over $G \in \operatorname{IFMG}(X)$ by $H \unlhd G$

Example 3.2. $\left(\mathrm{Z}_{4},+_{4}\right)$ is a group. Then

$$
G=\left\{\begin{array}{l}
<2:(0.6,0.4,0.3,0.1),(0.4,0.6,0.7,0.9)> \\
<1:(0.8,0.7,0.7,0.5,0.1,0.1),(0.2,0.3,0.3,0.5,0.9,0.9)>, \\
<3:(0.8,0.7,0.7,0.5,0.1,0.1),(0.2,0.3,0.3,0.5,0.9,0.9)>, \\
<0:(0.9,0.8,0.7,0.5,0.1,0.1),(0.1,0.8,0.3,0.5,0.9,0.9)>,
\end{array}\right.
$$

is an intuitionistic fuzzy multi group. And

$$
H=\left\{\begin{array}{l}
<2:(0.6,0.4,0.3,0.1),(0.4,0.6,0.7,0.9)> \\
<1:(0.7,0.6,0.5,0.5,0.1,0.1),(0.3,0.4,0.5,0.5,0.9,0.9)> \\
<3:(0.7,0.6,0.5,0.5,0.1,0.1),(0.3,0.4,0.5,0.5,0.9,0.9)> \\
<0:(0.9,0.8,0.7,0.5,0.1,0.1),(0.1,0.2,0.3,0.5,0.9,0.9)>
\end{array}\right\}
$$

is a normal sub-intuitionistic fuzzy multigroup of the intuitionistic fuzzy multigroup $G$.

Proposition 3.3. Let $G_{1}$ and $G_{2}$ be sub-intuitionistic fuzzymgroup of an intuitionistic fuzzy mgroup $G \in \operatorname{IFM} G(X)$ such that $G_{1}, G_{2} \in \Delta \wp \operatorname{SIFMG}(G)$, then 
I.M.Adamu, Y.Tella and A.J.Alkali

$\begin{array}{ll}\text { i. } & G_{1} \cap G_{2} \in \Delta \wp \operatorname{SIFMG}(G) \\ \text { ii. } & G_{1} \cup G_{2} \in \Delta \wp \operatorname{SIFMG}(G) .\end{array}$

Proof:

i. $\quad G_{1}$ and $G_{2}$ are sub-intuitionistic fuzzy mgroups, we have;

$$
\begin{gathered}
C M_{G_{1}}(x y) \geq C M_{G_{1}}(x) \wedge C M_{G_{1}}(y), C M_{G_{1}}\left(x^{-1}\right)=C M_{G_{1}}(x) \forall x, y \in X, \\
C M_{G_{2}}(x y) \geq C M_{G_{2}}(x) \wedge C M_{G_{2}}(y), C M_{G_{2}}\left(x^{-1}\right)=C M_{G_{2}}(x) \forall x, y \in X . \\
\therefore C M_{G_{1} \cap G_{2}}(x y)=\wedge\left\{C M_{G_{1}}(x y), C M_{G_{2}}(x y)\right\} \\
\geq \wedge\left\{\left[C_{G_{1}}(x) \wedge C_{G_{1}}(y)\right],\left[C_{G_{2}}(x) \wedge C_{G_{2}}(y)\right]\right\} \\
=C M_{G_{1}}(x) \wedge C M_{G_{1}}(y) \wedge C M_{G_{2}}(x) \wedge C M_{G_{2}}(y) \\
=\left[C M_{G_{1}}(x) \wedge C M_{G_{2}}(x)\right] \wedge\left[C M_{G_{1}}(y) \wedge C M_{G_{2}}(y)\right] \\
=C M_{G_{1} \cap G_{2}}(x) \wedge C M_{G_{1} \cap G_{2}}(y) \\
C M_{G_{1} \cap G_{2}}\left(x^{-1}\right)=C M_{G_{1}}\left(x^{-1}\right) \wedge C M_{G_{2}}\left(x^{-1}\right) \\
=C M_{G_{1}}(x) \wedge C M_{G_{2}}(x)
\end{gathered}
$$

Next we also have;

$C N_{G_{1}}(x y) \leq C N_{G_{1}}(x) \wedge C N_{G_{1}}(y), C N_{G_{1}}\left(x^{-1}\right)=C N_{G_{1}}(x) \forall x, y \in X$,

$C N_{G_{2}}(x y) \leq C N_{G_{2}}(x) \wedge C N_{G_{2}}(y), C N_{G_{2}}\left(x^{-1}\right)=C N_{G_{2}}(x) \forall x, y \in X$.$$
\therefore C N_{G_{1} \cap G_{2}}(x y)=\wedge\left\{C N_{G_{1}}(x y), C N_{G_{2}}(x y)\right\}
$$$$
\leq \mathrm{V}\left\{\left[C N_{G_{1}}(x) \wedge C N_{G_{1}}(y)\right],\left[C N_{G_{2}}(x) \wedge C N_{G_{2}}(y)\right]\right\}
$$$$
=C N_{G_{1}}(x) \wedge C N_{G_{1}}(y) \wedge C N_{G_{2}}(x) \wedge C N_{G_{2}}(y)
$$$$
=\left[C N_{G_{1}}(x) \wedge C N_{G_{2}}(x)\right] \wedge\left[C N_{G_{1}}(y) \wedge C N_{G_{2}}(y)\right]
$$

$=C N_{G_{1} \cap G_{2}}(x) \wedge C N_{G_{1} \cap G_{2}}(y)$

$$
\begin{gathered}
C N_{G_{1} \cap G_{2}}\left(x^{-1}\right)=C N_{G_{1}}\left(x^{-1}\right) \wedge C N_{G_{2}}\left(x^{-1}\right) \\
=C N_{G_{1}}(x) \wedge C N_{G_{2}}(x)
\end{gathered}
$$

$=C N_{G_{1} \cap G_{2}}(x)$

Thus, from (1), (2), (3) and (4), we have

$G_{1} \cap G_{2} \in \operatorname{IFMG}(X)$

Next

Since $G_{1} \subseteq G$ and $G_{2} \subseteq G$, then $G_{1} \cap G_{2} \subseteq G$

Since $G_{1}, G_{2} \in \triangle \wp S I F M G(M)$, we have

$C M_{G_{1}}\left(x^{-1} y_{1} x\right) \geq C M_{G_{1}}\left(y_{1}\right), \forall x \in G^{*}, y_{1} \in G_{1}{ }^{*}$;

$C M_{G_{2}}\left(x^{-1} y_{2} x\right) \geq C M_{G_{2}}\left(y_{2}\right), \forall x \in G^{*}, y_{2} \in G_{2}{ }^{*}$;

Now

$$
\begin{gathered}
C M_{G_{1} \cap G_{2}}\left(x^{-1} y x\right)=\wedge\left\{C M_{G_{1}}\left(x^{-1} y x\right), C M_{G_{2}}\left(x^{-1} y x\right)\right\} \\
\geq \wedge\left\{C M_{G_{1}}(y), C M_{G_{2}}(y)\right\} \forall x \in G^{*}, y \in\left(G_{1} \cap G_{2}\right)^{*}=G_{1}^{*} \cap G_{2}^{*}
\end{gathered}
$$

Since $C M_{G_{1}}\left(x^{-1} y_{1} x\right) \geq C M_{G_{1}}\left(y_{1}\right)$ and $C M_{G_{2}}\left(x^{-1} y_{2} x\right) \geq C M_{G_{2}}\left(y_{2}\right)$

$$
\therefore C M_{G_{1} \cap G_{2}}\left(x^{-1} y x\right) \geq C M_{G_{1}}(y) \wedge C M_{G_{2}}(y)=C M_{G_{1} \cap G_{2}}(y)
$$

Thus $C M_{G_{1} \cap G_{2}}\left(x^{-1} y x\right) \geq C M_{G_{1} \cap G_{2}}(y), \forall x \in G^{*}, y \in G_{1} \cap G_{2}$

Similarly,

$C N_{G_{1}}\left(x^{-1} y_{1} x\right) \leq C N_{G_{1}}\left(y_{1}\right), \forall x \in G^{*}, y_{1} \in G_{1}{ }^{*}$;

$C N_{G_{2}}\left(x^{-1} y_{2} x\right) \leq C N_{G_{2}}\left(y_{2}\right), \forall x \in G^{*}, y_{2} \in G_{2}{ }^{*}$; 
On Normal Sub-Intuitionistic Fuzzy Multigroups

Therefore,

$$
\begin{gathered}
C N_{G_{1} \cap G_{2}}\left(x^{-1} y x\right)=\vee\left\{C N_{G_{1}}\left(x^{-1} y x\right), C N_{G_{2}}\left(x^{-1} y x\right)\right\} \\
\leq \vee\left\{C N_{G_{1}}(y), C N_{G_{2}}(y)\right\} \forall x \in G^{*}, y \in\left(G_{1} \cap G_{2}\right)^{*}=G_{1}^{*} \cap G_{2}^{*} \\
\text { Since } C N_{G_{1}}\left(x^{-1} y_{1} x\right) \leq C N_{G_{1}}\left(y_{1}\right) \text { and } C N_{G_{2}}\left(x^{-1} y_{2} x\right) \leq C N_{G_{2}}\left(y_{2}\right) \\
\therefore C N_{G_{1} \cap G_{2}}\left(x^{-1} y x\right) \leq C N_{G_{1}}(y) \wedge C N_{G_{2}}(y)=N(y)
\end{gathered}
$$

Thus $C N_{G_{1} \cap G_{2}}\left(x^{-1} y x\right) \leq C N_{G_{1} \cap G_{2}}(y), \forall x \in G^{*}, y \in G_{1} \cap G_{2}$

From equations (5), (6) and (7) we have, $G_{1} \cap G_{2} \in \Delta \wp S I F M G(M)$.

ii. Since $G_{1}$ and $G_{2}$ are sub-intuitionistic fuzzy mgroups, we have;

$$
\begin{gathered}
C M_{G_{1}}(x y) \geq C M_{G_{1}}(x) \wedge C M_{G_{1}}(y), C M_{G_{1}}\left(x^{-1}\right)=C M_{G_{1}}(x) \forall x, y \in X, \\
C M_{G_{2}}(x y) \geq C M_{G_{2}}(x) \wedge C M_{G_{2}}(y), C M_{G_{2}}\left(x^{-1}\right)=C M_{G_{2}}(x) \forall x, y \in X . \\
\therefore C M_{G_{1} \cup G_{2}}(x y)=\vee\left\{C M_{G_{1}}(x y), C M_{G_{2}}(x y)\right\} \\
\geq \vee\left\{\left[C M_{G_{1}}(x) \wedge C M_{G_{1}}(y)\right],\left[C M_{G_{2}}(x) \wedge C M_{G_{2}}(y)\right]\right\} \\
=\left[C M_{G_{1}}(x) \wedge C M_{G_{2}}(x)\right] \vee\left[C M_{G_{1}}(y) \wedge C M_{G_{2}}(y)\right] \\
=\left[C M_{G_{1}}(x) \vee C M_{G_{2}}(x)\right] \wedge\left[C M_{G_{1}}(y) \vee C M_{G_{2}}(y)\right] \\
=C M_{G_{1} \cup G_{2}}(x) \wedge C M_{G_{1} \cup G_{2}}(y) \\
C M_{G_{1} \cup G_{2}}\left(x^{-1}\right)=C M_{G_{1}}\left(x^{-1}\right) \vee C M_{G_{2}}\left(x^{-1}\right) \\
=C_{M_{1}}(x) \vee C_{M_{2}}(x)=C_{M_{1} \cup M_{2}}(x) \\
\therefore C M_{G_{1} \cup G_{2}}\left(x^{-1}\right)=C M_{G_{1} \cup G_{2}}(x)
\end{gathered}
$$

We also have;

$$
\begin{gathered}
C N_{G_{1}}(x y) \geq C N_{G_{1}}(x) \wedge C N_{G_{1}}(y), C N_{G_{1}}\left(x^{-1}\right)=C N_{G_{1}}(x) \forall x, y \in X, \\
C N_{G_{2}}(x y) \geq C N_{G_{2}}(x) \wedge C N_{G_{2}}(y), C N_{G_{2}}\left(x^{-1}\right)=C N_{G_{2}}(x) \forall x, y \in X . \\
\therefore C N_{G_{1} \cup G_{2}}(x y)=\vee\left\{C N_{G_{1}}(x y), C N_{G_{2}}(x y)\right\} \\
\leq \vee\left\{\left[C N_{G_{1}}(x) \wedge C N_{G_{1}}(y)\right],\left[C N_{G_{2}}(x) \wedge C N_{G_{2}}(y)\right]\right\} \\
=\left[C N_{G_{1}}(x) \wedge C N_{G_{2}}(x)\right] \vee\left[C N_{G_{1}}(y) \wedge C N_{G_{2}}(y)\right] \\
=\left[C N_{G_{1}}(x) \vee C N_{G_{2}}(x)\right] \wedge\left[C N_{G_{1}}(y) \vee C N_{G_{2}}(y)\right] \\
=C N_{G_{1} \cup G_{2}}(x) \wedge C N_{G_{1} \cup G_{2}}(y) \\
C N_{G_{1} \cup G_{2}}\left(x^{-1}\right)=C N_{G_{1}}\left(x^{-1}\right) \vee C N_{G_{2}}\left(x^{-1}\right) \\
=C N_{G_{1}}(x) \vee C N_{G_{2}}(x) \\
=C N_{G_{1} \cup G_{2}}(x)
\end{gathered}
$$

Thus, from (1), (2), (3) and (4), we have

Next

$$
G_{1} \cup G_{2} \in \operatorname{IFMGMG}(X)
$$

Since $G_{1} \subseteq G$ and $G_{2} \subseteq G$, then $G_{1} \cup G_{2} \subseteq G$

Since $G_{1}, G_{2} \in \triangle \wp \operatorname{SIFMG}(M)$, we have

$$
\begin{aligned}
& C M_{G_{1}}\left(x^{-1} y_{1} x\right) \geq C M_{G_{1}}\left(y_{1}\right), \forall x \in G^{*}, y_{1} \in G_{1}{ }^{*} \text {; } \\
& C M_{G_{2}}\left(x^{-1} y_{2} x\right) \geq C M_{G_{2}}\left(y_{2}\right), \forall x \in G^{*}, y_{2} \in G_{2}{ }^{*} \text {; } \\
& \therefore C_{M_{1} \cup M_{2}}\left(x^{-1} y x\right)=\mathrm{V}\left\{C_{M_{1}}\left(x^{-1} y x\right), C_{M_{2}}\left(x^{-1} y x\right)\right\} \\
& \geq \vee\left\{C M_{G_{1}}(y), C M_{G_{2}}(y)\right\} \forall x \in G^{*}, y \in\left(G_{1} \cup G_{2}\right)^{*}=G_{1}^{*} \cup G_{2}^{*}
\end{aligned}
$$


I.M.Adamu, Y.Tella and A.J.Alkali

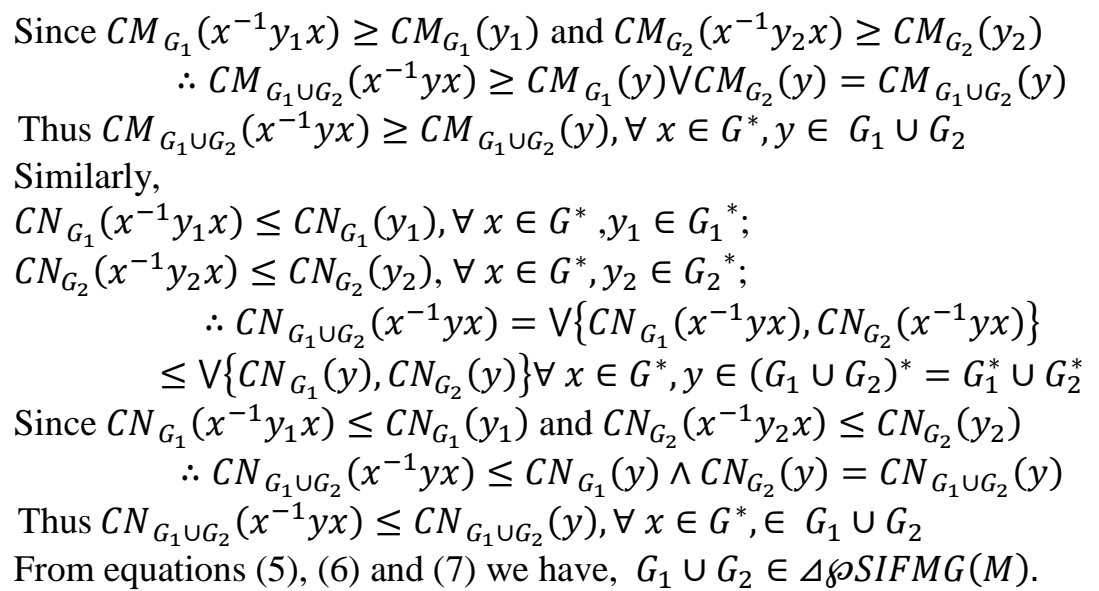

Remark 3.4. Let $\left\{G_{i}: G_{i} \in \Delta \wp S I F M G(M), i \in \Delta\right\}$ then $\bigcap_{i \in \Delta} G_{i} \in \Delta \wp S I F M G(M)$ and $\bigcup_{i \in \Delta} M_{i} \in \Delta \wp(M)$.

Proposition 3.5. If $H \in \triangle \wp \operatorname{SIFMG}(G)$ then $H^{*}$ is a normal sub-intuitionistic group of $G^{*}$.

Proof:

Since $H \subseteq G \Rightarrow H^{*} \subseteq G^{*}$

Thus, $H^{*}$ is a sub-intuitionistic group of $G^{*}$

Since $H \in \triangle \wp \operatorname{SIFMG}(G)$, then for any $x \in G^{*}$ and $h \in H^{*}$

$C M_{H}\left(x^{-1} h x\right) \geq C M_{H}(h)>0$

$\therefore C M_{H}\left(x^{-1} h x\right)>0$

Similarly,

$C N_{H}\left(x^{-1} h x\right) \leq C N_{H}(h)=0$

$\therefore C M_{H}\left(x^{-1} h x\right)=0$

$\Rightarrow\left(x^{-1} h x\right) \in H^{*}$

$\therefore H^{*}$ is a normal sub-intuitionistic group of $G^{*}$.

Proposition 3.6. Let $G \in F M G(X)$ and $H \in \triangle \wp S I F M G(G)$. Then $H^{j *}$ is a normal subgroup of $G^{*}$ iff

$$
\mu_{H}^{j+1}\left(x^{-1} y x\right)=0 \text { and } v_{H}^{j+1}\left(x^{-1} y x\right)=0 \forall x \in G^{*} \text { and } y \in H^{j *} \text {. }
$$

Proof: Let $x \in G^{*}$ and $y \in H^{j *}$. It implies that

$\mu_{H}^{j}\left(x^{-1} y x\right) \geq 0$ And $\mu_{H}^{j+1}\left(x^{-1} y x\right)=0$ (by definition 2.19)

$v_{H}^{j}\left(x^{-1} y x\right)=0$ and $v_{H}^{j+1}\left(x^{-1} y x\right)=0$

Assume $\mu_{H}^{j+1}\left(x^{-1} y x\right)=0$ and $v_{H}^{j+1}\left(x^{-1} y x\right)=0 \forall x \in G^{*}$ and $y \in H^{j *}$

Then by the above proposition,

$\mu_{H}^{j}\left(x^{-1} y x\right)>0$ and $v_{H}^{j}\left(x^{-1} y x\right)=0$

Conversely,

$\Longrightarrow x^{-1} y x \in H^{j *}$. Then $H^{j *}$ is a normal subgroup of $G^{*}$. Hence the proof

$H^{j *}$ is a normal subgroup of $G^{*}$. Then $x \in G^{*}, y \in H^{j *} \Rightarrow x^{-1} y x \in H^{j *}$

$\Rightarrow \mu_{H}^{j+1}\left(x^{-1} y x\right)=0$ and $v_{H}^{j+1}\left(x^{-1} y x\right)=0$. Hence the proof. 
On Normal Sub-Intuitionistic Fuzzy Multigroups

Proposition 3.7. Let $X$ be a group and $G \in \operatorname{IFM} G(X)$. If $G \in \triangle \wp S I F M G(G)$, then $G^{-1} \in \triangle \wp \operatorname{SIFMG}(G)$.

Proof: Since $G \in \triangle \wp \operatorname{SIFMG}(G)$, then we have $C M_{M}\left(x^{-1} y x\right) \geq C M_{M}(y), \forall x \in X$ and $y \in G^{*}$

Also

$$
\begin{gathered}
C M_{G^{-1}}(x y)=C M_{G}\left[(x y)^{-1}\right]=C M_{G}(x y) \geq C M_{G}(x) \wedge C M_{G}(y) \\
=C M_{G}\left(x^{-1}\right) \wedge C M_{G}\left(y^{-1}\right) \\
=C M_{G^{-1}}(x) \wedge C M_{G^{-1}}(y) \\
\therefore C M_{G^{-1}}(x y) \geq C M_{G}\left(x^{-1}\right) \wedge C M_{G}\left(y^{-1}\right) \\
C M_{G^{-1}}\left(x^{-1}\right)=C M_{G}\left[\left(x^{-1}\right)^{-1}\right]=C M_{G}(x) \\
=C M_{G}\left(x^{-1}\right)=C M_{G^{-1}}(x) \\
\therefore C M_{G^{-1}}\left(x^{-1}\right)=C M_{G^{-1}}(x)
\end{gathered}
$$

Thus, $G^{-1} \in \operatorname{IFMG}(X)$

$$
C M_{G^{-1}}\left(x^{-1} y x\right)=C M_{G}\left[\left(x^{-1} y x\right)^{-1}\right]=C M_{G}\left(x^{-1} y x\right) \geq C M_{G}(y), \forall x \in X
$$

And $y \in G^{*}$

We also have;

$$
\Rightarrow C M_{G^{-1}}\left(x^{-1} y x\right) \geq C M_{G^{-1}}(y)(1)
$$

$$
\begin{gathered}
\therefore C M_{G^{-1}}\left(x^{-1} y x\right)=C M_{G}\left[\left(x^{-1} y x\right)^{-1}\right] \\
=C M_{G}\left(x^{-1} y x\right) \geq C M_{G}(y)=C M_{G^{-1}}(y)
\end{gathered}
$$$$
C N_{G}\left(x^{-1} y x\right) \geq C N_{G}(y), \forall x \in X \text { and } y \in G^{*}
$$

And

$$
\begin{aligned}
& C N_{G^{-1}}(x y)=C N_{G}\left[(x y)^{-1}\right]=C N_{G}(x y) \geq C N_{G}(x) \wedge C N_{G}(y) \\
& =C N_{G}\left(x^{-1}\right) \wedge C N_{G}\left(y^{-1}\right) \\
& =C N_{G^{-1}}(x) \wedge C N_{G^{-1}}(y) \\
& \therefore C N_{G^{-1}}(x y) \geq C N_{G}\left(x^{-1}\right) \wedge C N_{G}\left(y^{-1}\right) \\
& C N_{G^{-1}}\left(x^{-1}\right)=C N_{G}\left[\left(x^{-1}\right)^{-1}\right]=C N_{G}(x) \\
& =C N_{G}\left(x^{-1}\right)=C N_{G^{-1}}(x) \\
& \therefore C N_{G^{-1}}\left(x^{-1}\right)=C N_{G^{-1}}(x) \\
& \text { Thus, } G^{-1} \in \operatorname{IFMG}(X) \\
& C N_{G^{-1}}\left(x^{-1} y x\right)=C N_{G}\left[\left(x^{-1} y x\right)^{-1}\right]=C N_{G}\left(x^{-1} y x\right) \geq C N_{G}(y), \forall x \in X \\
& \text { and } y \in G^{*} \\
& \Rightarrow C N_{G^{-1}}\left(x^{-1} y x\right) \geq C N_{G^{-1}}(y)(2) \\
& \therefore C N_{G^{-1}}\left(x^{-1} y x\right)=N\left[\left(x^{-1} y x\right)^{-1}\right] \\
& =C N_{G}\left(x^{-1} y x\right) \geq C N_{G}(y)=C N_{G^{-1}}(y) \\
& \text { Hence from (1) and (2) we have } G^{-1} \in \triangle \wp \operatorname{SIFMG}(G)
\end{aligned}
$$

Proposition 3.8. Let $X, Y$ be two groups and $f: X \rightarrow Y$ be an isomorphism of groups. Suppose $G_{1} \in \operatorname{IFMG}(X), G_{2} \in \operatorname{IFMG}(Y)$ and $f\left(G_{1}\right) \subseteq G_{2}$

i. if $H \in \triangle \wp S I F M G\left(G_{1}\right)$, then $f(H) \in \triangle \wp \operatorname{SIFMG}\left(G_{2}\right)$.

ii. if $H \in \triangle \wp S I F M G\left(G_{2}\right)$ then $f^{-1}(H) \in \triangle \wp S I F M G\left(G_{1}\right)$.

Proof: i. Let $H \in \triangle \wp \operatorname{SIFMG}\left(G_{1}\right)$. Clearly $H$ is a sub-intuitionistic fuzzy mgroup of $G_{1}$ (by definition).

We show that $f(H)$ is a sub-intuitionistic fuzzy of $G_{2}$. 
I.M.Adamu, Y.Tella and A.J.Alkali

$C M_{f(H)}(x y)=\mathrm{V}_{f(z)=x y} C M_{H}(z)$ Since $f^{-1}(x y) \neq \emptyset$ (by definition 2.9. (i))

But $f$ is $1-1$ and onto (by hypothesis), thus $f^{-1}(x y)=\{z\}$.

In particular,

$C M_{f(H)}(x y)=C M_{H}(z)=C M_{H}\left(f^{-1}(x y)\right)=C M_{H}\left(f^{-1}(x) f^{-1}(y)\right)$

( $f$ is an isomorphism). But

$C M_{H}\left(f^{-1}(x) f^{-1}(y)\right) \geq C M_{H}\left(f^{-1}(x)\right) \wedge C M_{H}\left(f^{-1}(y)\right)$

(His submgroup of $M_{1}$ ). But

$C M_{H}\left(f^{-1}(x)\right) \wedge C M_{H}\left(f^{-1}(y)\right)=C M_{\left(f^{-1}\right)^{-1}(H)}(x) \wedge C M_{\left(f^{-1}\right)^{-1}(H)}(y)$

(by definition 2.9.(ii)) and

$C M_{\left(f^{-1}\right)^{-1}(H)}(x) \wedge C M_{\left(f^{-1}\right)^{-1}(H)}(y)=C M_{f(H)}(x) \wedge C M_{f(H)}(y)$

Thus using (1), (2), (3) and (4), we have:

$C M_{f(H)}(x y) \geq C M_{f(H)}(x) \wedge C M_{f(H)}(y)$

$C M_{f(H)}\left(x^{-1}\right)=\vee_{f(y)=x^{-1}} C M_{H}(y)$ (Since $f^{-1}\left(x^{-1}\right) \neq \varnothing$

(by definition 2.9.(i))

But $f$ is $1-1$ and onto (by hypothesis), thus $f^{-1}\left(x^{-1}\right)=\{y\}$.

In particular,

$C M_{f(H)}\left(x^{-1}\right)=C M_{H}(y)=C M_{H}\left(f^{-1}\left(x^{-1}\right)\right)=C M_{H}\left(\left(f^{-1}(x)\right)^{-1}\right)$

But

$C M_{H}\left(\left(f^{-1}(x)\right)^{-1}\right) \geq C M_{H}\left(f^{-1}(x)\right)=C M_{\left(f^{-1}\right)^{-1}(H)}(x)=C M_{f(H)}(x)$

Thus from (6) and (7) we have

$C M_{f(H)}\left(x^{-1}\right) \geq C M_{f(H)}(x)$

Also

$C N_{f(H)}(x y)=C N_{H}(z)=C N_{H}\left(f^{-1}(x y)\right)=C N_{H}\left(f^{-1}(x) f^{-1}(y)\right)$

( $f$ is an isomorphism). But

$C N_{H}\left(f^{-1}(x) f^{-1}(y)\right) \leq C N_{H}\left(f^{-1}(x)\right) \wedge C N_{H}\left(f^{-1}(y)\right)$

(His submgroup of $M_{1}$ ). But

$C N_{H}\left(f^{-1}(x)\right) \wedge C N_{H}\left(f^{-1}(y)\right)=C N_{\left(f^{-1}\right)^{-1}(H)}(x) \wedge C N_{\left(f^{-1}\right)^{-1}(H)}(y)$

(by definition 2.9.(ii)) and

$C N_{\left(f^{-1}\right)^{-1}(H)}(x) \wedge C N_{\left(f^{-1}\right)^{-1}(H)}(y)=C N_{f(H)}(x) \wedge C N_{f(H)}(y)$

Thus using (9), (10), (11) and (12), we have:

$$
\begin{aligned}
& C N_{f(H)}(x y) \leq C N_{f(H)}(x) \wedge C N_{f(H)}(y) \\
& C N_{f(H)}\left(x^{-1}\right)=C N_{H}(y)=C N_{H}\left(f^{-1}\left(x^{-1}\right)\right)=C N_{H}\left(\left(f^{-1}(x)\right)^{-1}\right)
\end{aligned}
$$

But $C N_{H}\left(\left(f^{-1}(x)\right)^{-1}\right) \leq C M_{H}\left(f^{-1}(x)\right)=C N_{\left(f^{-1}\right)^{-1}(H)}(x)=C N_{f(H)}(x)$

Thus from (14) and (15) we have

$C N_{f(H)}\left(x^{-1}\right) \leq C N_{f(H)}(x)$

From (5), (8),(13) and (16) we have $f(H) \in I F M G(Y)$

But $f\left(G_{1}\right) \subseteq G_{2}$, since $H \subseteq G_{1}$, then $f(H) \subseteq G_{2}$

Hence $f(H)$ is a sub-intuitionistic mgroup of $G_{2}$

Now we show that $f(H) \unlhd G_{2}$. 
On Normal Sub-Intuitionistic Fuzzy Multigroups

i.e. $C M_{f(H)}\left(x^{-1} y x\right) \geq C M_{f(H)}(y)$ for all $x \in G_{2}^{*}$ and $y \in(f(H))^{*}$ and $C N_{f(H)}\left(x^{-1} y x\right) \leq C M_{f(H)}(y)$ for all $x \in G_{2}^{*}$ and $y \in(f(H))^{*}$

Now

$C M_{f(H)}\left(x^{-1} y x\right)=\mathrm{V}_{f(z)=x^{-1} y x} C M_{H}(z)=C M_{H}\left(f^{-1}\left(x^{-1} y x\right)\right)$

(Since $f$ is $1-1$ and onto by definition)

But $C M_{H}\left(f^{-1}\left(x^{-1} y x\right)\right)=C M_{H}\left(f^{-1}\left(x^{-1}\right) f^{-1}(y) f^{-1}(x)\right)$

and

$C M_{H}\left(f^{-1}\left(x^{-1}\right) f^{-1}(y) f^{-1}(x)\right)=C M_{H}\left(\left(f^{-1}(x)\right)^{-1} f^{-1}(y) f^{-1}(x)\right)$

(since $f$ is an isomorphism by hypothesis)

But

$C M_{H}\left(\left(f^{-1}(x)\right)^{-1} f^{-1}(y) f^{-1}(x)\right) \geq C M_{H}\left(f^{-1}(y)\right)=C M_{f(H)}(y)$

From (19), (20) and (21), we have

$C M_{f(H)}\left(x^{-1} y x\right) \geq C M_{f(H)}(y)$

Similarly,

$C N_{f(H)}\left(x^{-1} y x\right)=\mathrm{V}_{f(z)=x^{-1} y x} C N_{H}(z)=C N_{H}\left(f^{-1}\left(x^{-1} y x\right)\right)$

(Since $f$ is $1-1$ and onto by definition)

But $C N_{H}\left(f^{-1}\left(x^{-1} y x\right)\right)=C N_{H}\left(f^{-1}\left(x^{-1}\right) f^{-1}(y) f^{-1}(x)\right)$

and

$$
C N_{H}\left(f^{-1}\left(x^{-1}\right) f^{-1}(y) f^{-1}(x)\right)=C N_{H}\left(\left(f^{-1}(x)\right)^{-1} f^{-1}(y) f^{-1}(x)\right)
$$

(since $f$ is an isomorphism by hypothesis)

But

$$
C N_{H}\left(\left(f^{-1}(x)\right)^{-1} f^{-1}(y) f^{-1}(x)\right) \leq C N_{H}\left(f^{-1}(y)\right)=C N_{f(H)}(y)
$$

From (23), (24) and (25), we have

$$
C N_{f(H)}\left(x^{-1} y x\right) \leq C N_{f(H)}(y)
$$

Hence, using (18), (22) and (26) we deduce that $f(H) \in \triangle \wp S I F M G\left(G_{2}\right)$

ii. Let $H$ be a sub-intuitionistic fuzzy mgroup of $G_{2}$.

We show that $f^{-1}(H)$ is a sub-intuitionisticfuzzy mgroup of $G_{1}$.

But $C M_{f^{-1}(H)}(x y)=C M_{H}[f(x y)]$ (by definition)

$=C M_{H}[f(x) f(y)](f$ is an isomorphism)

$\geq C M_{H}[f(x)] \wedge C M_{H}[f(y)]$ (by definition)

$$
=C M_{f^{-1}(H)}(x) \wedge C M_{f^{-1}(H)}(y)
$$

$\therefore C M_{f^{-1}(H)}(x y) \geq C M_{f^{-1}(H)}(x) \wedge C M_{f^{-1}(H)}(y)$

$C M_{f^{-1}(H)}\left(x^{-1}\right)=C M_{H}\left[f\left(x^{-1}\right)\right]$ (by definition)

$=C M_{H}\left[(f(x))^{-1}\right](f$ is an isomorphism $)$

$=C M_{H}[f(x)]($ His a submgroup)

$=C M_{f^{-1}(H)}(x)$ (by definition)

$$
\text { Thus } C M_{f^{-1}(H)}\left(x^{-1}\right)=C M_{f^{-1}(H)}(x)
$$

$C N_{f^{-1}(H)}(x y)=C N_{H}[f(x y)]=C N_{H}[f(x) f(y)](f$ is an isomorphism $)$ 
I.M.Adamu, Y.Tella and A.J.Alkali

$$
\begin{aligned}
& \leq C N_{H}[f(x)] \wedge C N_{H}[f(y)] \text { (by definition) } \\
& \quad=C N_{f^{-1}(H)}(x) \wedge C N_{f^{-1}(H)}(y) \\
& \quad \therefore C N_{f^{-1}(H)}(x y) \quad \leq C N_{f^{-1}(H)}(x) \wedge C N_{f^{-1}(H)}(y) \\
& C N_{f^{-1}(H)}\left(x^{-1}\right)=C N_{H}\left[f\left(x^{-1}\right)\right] \text { (by definition) } \\
& =C N_{H}\left[(f(x))^{-1}\right](f \text { is an isomorphism) } \\
& =C N_{H}[f(x)](H \text { is a submgroup) } \\
& =C N_{f^{-1}(H)}(x) \text { (by definition) } \\
& \text { Thus } C N_{f^{-1}(H)}\left(x^{-1}\right)=C N_{f^{-1}(H)}(x) \\
& \text { Now for all } x \in M_{1}^{*} \text { and } y \in\left[f^{-1}(H)\right]^{*} \text { we have } \\
& C M_{f^{-1}(H)}\left(x^{-1} y x\right)=C M_{H}\left[f\left(x^{-1} y x\right)\right] \text { (by definition) } \\
& =C M_{H}\left[(f(x))^{-1} f(y) f(x)\right](f \text { is an isomorphism) } \\
& \geq C M_{H}[f(y)]\left(H \in \Delta \wp S I F M G\left(G_{2}\right)\right. \text { by hypothesis) } \\
& =C M_{f^{-1}(H)}(y)(\text { by definition) } \\
& \quad \therefore C M_{f^{-1}(H)}\left(x^{-1} y x\right) \geq C M_{f^{-1}(H)}(y) \\
& C M_{f^{-1}(H)}\left(x^{-1} y x\right)=C M_{H}\left[f\left(x^{-1} y x\right)\right] \text { (by definition) } \\
& =C N_{H}\left[(f(x))^{-1} f(y) f(x)\right](f \text { is an isomorphism) } \\
& \leq C M_{H}[f(y)]\left(H \in \Delta \wp S I F M G\left(G_{2}\right)\right. \text { by hypothesis) } \\
& =C N_{f^{-1}(H)}(y)(\text { by definition) } \\
& \therefore C N_{f^{-1}(H)}\left(x^{-1} y x\right) \leq C N_{f^{-1}(H)}(y)(6)
\end{aligned}
$$

Hence from (27) to (31) we have $f^{-1}(H)$ is a normal subintuitionistic fuzzy mgroup of $G_{1}$.

\section{Conclusion}

The paper developed the concept of normal sub-intuitionistic fuzzy Multigroup with some of its related algebraic structures such as intersection, union, of any two normal sub-intuitionistic fuzzy multigroups are also normal. It also showed that the inverse of any normal sub-intuitionistic fuzzy multigroup is normal and for any normal subintuitionistic multigroup, the root (support) set of normal sub-intuitionistic fuzzy multigroup is a normal sub-intuitionistic fuzzy group. Finally, it showed that under the isomorphism function between any two groups, the image of a normal sub-intuitionistic fuzzy multigroup under the isomorphism is a normal sub-intuitionistic fuzzy multigroup and the inverse image of a normal sub-intuitionistic fuzzy multigroup under the isomorphism is a normal sub-intuitionistic fuzzy multigroup.

\section{REFERENCES}

1. W.D.Blizard, Dedekind multisets and functions shells, Theoret. Computer Science, 110 (1993) 79-98.

2. W.D.Blizard, Multiset theory, Notre Dame J. Form. Log., 30(1) (1989) 36-66.

3. A.Asyropoulos, Mathematics of Multisets, C.S.Calude et al. (Eds.): Multiset Processing, LNCS, 347-358, 2001.

4. A.Asyropoulos, Categorical modeels of multisets, Romanain Journal of Information Science and Technology, 6(3-4) (2003) 393-400.

5. L.A.Zadeh, Fuzzy sets, Information and Control, 8 (1965) 338-353. 
On Normal Sub-Intuitionistic Fuzzy Multigroups

6. K.T.Atanassov, Intuitionistic fuzzy sets, VII ITKRs Session,Sofia (deposed inCentral Science-Technical Library of BulgarianAccademy of Science, 1697/84), 1983(in Bulgarian)

7. K.T.Atannassov, Intuitionistic fuzzy sets, Fuzzy Sets and Systems, 20 (1986) 87-96.

8. G.Takeuti and S.Ttani, Intuitionistic fuzzy logic and intuitionistic fuzzy set theory, $J$. Symbolic Logic, 49 (1984) 851-866.

9. R.R.Yager, On theory of bags, Intertional Journal of General Systems, 13 (1986) 2327.

10. T.K.Shinoj and J.J.Sunil, Intuitionistic fuzzy multisets, International Journal Engineering Science and Innovative Technology, 2(6) (2013) 1-24.

11. T.K.Shinoj, A.Baby and J.J.Sunil, On some algebraic structures of fuzzy multisets, Annals of Fuzzy Mathematics and Informatics, 9(1) (2015) 77-90.

12. I.M.Adamu, Y.Tella and A.J.Alkali, On normal and soft normal groups under multisets and soft multisets context, MAYFEB Journal of Mathematics, 2(2017) 34-4.

13. S.P.Jena, S.K.Ghosh and B.K.Tripathi, On theory of bags and lists, Information Sciences, 132 (2011) 241-254.

14. S.K.Nazmul, P.Majumdar and S.K.Samanta, On multisets and multigroups, Annals Fuzzy Mathematics and Informatics, 6(3) (2013) 643-656.

15. B.Sumita, Classical Sets and Non-Classical Sets: An Overview, Resonance, 10(8) (2005).

16. J.N.Mordeson, K.R.Bhutani and A.Rosenfeld, Fuzzy Group Theory, Springer, 2005.

17. T.K.Shinoj and S.J.John, Intuitionistic fuzzy multigroups, Annals of Pure and Applied Mathematics, 9(1) (2015) 131-143. 